Supplement of Clim. Past, 17, 2223-2254, 2021

https://doi.org/10.5194/cp-17-2223-2021-supplement

(c) Author(s) 2021. CC BY 4.0 License.

(c) (1)

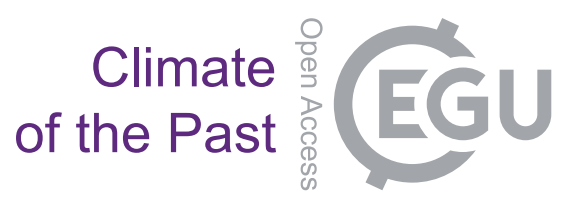

Supplement of

\title{
Data-constrained assessment of ocean circulation changes since the middle Miocene in an Earth system model
}

Katherine A. Crichton et al.

Correspondence to: Katherine A. Crichton (k.a.crichton@exeter.ac.uk)

The copyright of individual parts of the supplement might differ from the article licence. 


\section{Supplementary material A}

\section{Surface ocean temperatures}

\begin{tabular}{|c|c|c|c|c|c|c|c|c|}
\hline Age (Ma) & Longitude & Latitude & $T(\operatorname{deg} C)$ & Site name & Reference & $\begin{array}{l}\text { Paleo } \\
\text { Latitude }\end{array}$ & $\begin{array}{l}\text { Paleo } \\
\text { longitude }\end{array}$ & Proxy \\
\hline 0.006 & 1775.0 & \begin{tabular}{c|c|}
-46.0 \\
\end{tabular} & 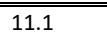 & 2594 & Herbert et al 2016 & 175.00 & $\begin{array}{l}-46.00 \\
\end{array}$ & Alkenone \\
\hline 0.006 & 60.0 & 17.0 & 26.8 & 722 & Herbert et al 2016 & 60.00 & 17.00 & Alkenone \\
\hline 0.006 & 159.0 & 0.0 & 27 & 806 & Zhang et al 2014 & 159.00 & 0.00 & TEX86 \& Alk \\
\hline 0.006 & -91.0 & -3.0 & 23 & 846 & Zhang et al 2014 & -91.00 & -3.00 & Alkenone \\
\hline 0.006 & -95.0 & 0.0 & 22.5 & 847 & Zhang et al 2014 & -95.00 & 0.00 & Alkenone \\
\hline 0.006 & -111.0 & 1.0 & 25.2 & 850 & Herbert et al 2016 & -111.00 & 1.00 & Alkenone \\
\hline 0.006 & -111.0 & 1.0 & 22 & 850 & Zhang et al 2014 & -111.00 & 1.00 & TEX86 \& Alk \\
\hline 0.006 & -148.0 & 54.0 & 7.2 & 887 & Herbert et al 2016 & -148.00 & 54.00 & Alkenone \\
\hline 0.006 & -13.0 & 69.0 & 1.77 & 907 & Herbert et al 2016 & -13.00 & 69.00 & Alkenone \\
\hline 0.006 & -16.0 & 58.0 & 10.8 & 982 & Herbert et al 2016 & -16.00 & 58.00 & Alkenone \\
\hline 0.006 & -118.0 & 30.0 & 17.3 & 1010 & Herbert et al 2016 & -118.00 & 30.00 & Alkenone \\
\hline 0.006 & -128.0 & 39.0 & 14.32 & 1021 & Herbert et al 2016 & -128.00 & 39.00 & Alkenone \\
\hline 0.006 & 14.0 & -30.0 & 17.33 & 1085 & Herbert et al 2016 & 14.00 & -30.00 & Alkenone \\
\hline 0.006 & 14.0 & -41.0 & 13.8 & 1088 & Herbert et al 2016 & 14.00 & -41.00 & Alkenone \\
\hline 0.006 & -178.0 & -42.0 & 14.45 & 1125 & Herbert et al 2016 & -178.00 & -42.00 & Alkenone \\
\hline 0.006 & 158.5 & 36.1 & 18.7 & 1208 & Herbert et al 2016 & 158.50 & 36.10 & Alkenone \\
\hline 0.006 & -86.0 & 6.0 & 27.73 & 1241 & Herbert et al 2016 & -86.00 & 6.00 & Alkenone \\
\hline 0.006 & -118.0 & 3.0 & 25.6 & U1338 & Herbert et al 2016 & -118.00 & 3.00 & Alkenone \\
\hline 0.006 & 168.0 & 51.0 & 5.4 & $883 / 4$ & Herbert et al 2016 & 168.00 & 51.00 & Alkenone \\
\hline 0.006 & 14.0 & 44.0 & 19.2 & med & Herbert et al 2016 & 14.00 & 44.00 & Alkenone \\
\hline 2.5 & $\begin{array}{l}60.0 \\
\end{array}$ & 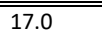 & 27.5 & $\overline{7722}$ & "Herbert et al 2016 & 259.49 & 16.30 & Alkenone \\
\hline 2.5 & 121.0 & 9.0 & 29 & 769 & Zhang et al 2014 & 120.62 & 9.05 & Alkenone \\
\hline 2.5 & 159.0 & 0.0 & 27.5 & 806 & Zhang et al 2014 & 160.74 & -0.94 & TEX86 \& Alk \\
\hline 2.5 & -91.0 & -3.0 & 25 & 846 & Zhang et al 2014 & -92.32 & -3.19 & Alkenone \\
\hline 2.5 & -95.0 & 0.0 & 25 & 847 & Zhang et al 2014 & $\begin{array}{l}-96.38 \\
\end{array}$ & -0.13 & Alkenone \\
\hline 2.5 & -111.0 & 1.0 & 25.5 & 850 & Herbert et al 2016 & -109.26 & 0.45 & Alkenone \\
\hline 2.5 & -111.0 & 1.0 & 24.5 & 850 & Zhang et al 2014 & -109.26 & 0.45 & Alkenone \\
\hline 2.5 & -13.0 & 69.0 & 6 & 907 & Herbert et al 2016 & -13.50 & 69.02 & Alkenone \\
\hline 2.5 & -16.0 & 58.0 & 14 & 982 & Herbert et al 2016 & -15.64 & 58.00 & Alkenone \\
\hline 2.5 & -118.0 & 30.0 & 18 & 1010 & Herbert et al 2016 & -116.74 & 29.30 & Alkenone \\
\hline 2.5 & -128.0 & 39.0 & 13 & 1021 & Herbert et al 2016 & -126.83 & 38.16 & Alkenone \\
\hline 2.5 & 14.0 & -30.0 & 19 & 1085 & Herbert et al 2016 & 13.50 & -30.17 & Alkenone \\
\hline 2.5 & 14.0 & -41.0 & 18 & 1088 & Herbert et al 2016 & 13.45 & -41.17 & Alkenone \\
\hline
\end{tabular}




\begin{tabular}{|c|c|c|c|c|c|c|c|c|}
\hline Age (Ma) & Longitude & Latitude & $T(\operatorname{deg} C)$ & Site name & Reference & $\begin{array}{l}\text { Paleo } \\
\text { Latitude }\end{array}$ & $\begin{array}{l}\text { Paleo } \\
\text { longitude }\end{array}$ & Proxy \\
\hline 2.5 & "-178.0 & -42.0 & 18 & 1125 & "Herbert et al 2016 & -176.41 & -43.09 & Alkenone \\
\hline 2.5 & 113.0 & 9.0 & 28 & 1143 & Zhang et al 2014 & 112.64 & 9.04 & Alkenone \\
\hline 2.5 & 158.5 & 36.1 & 21 & 1208 & Herbert et al 2016 & 160.67 & 35.15 & Alkenone \\
\hline 2.5 & -86.0 & 6.0 & 27.9 & 1241 & Herbert et al 2016 & -87.19 & 4.38 & Alkenone \\
\hline 2.5 & -118.0 & 3.0 & 27 & U1338 & Herbert et al 2016 & -116.20 & 1.80 & Alkenone \\
\hline 2.5 & -118.0 & 3.0 & 26 & U1338 & Zhang et al 2014 & -116.20 & 1.80 & Alkenone \\
\hline 2.5 & 168.0 & 51.0 & 7 & $883 / 4$ & Herbert et al 2016 & 170.26 & 49.98 & Alkenone \\
\hline 2.5 & 14.0 & 44.0 & 25 & med & Herbert et al 2016 & 13.76 & 43.83 & Alkenone \\
\hline 4.5 & 175.0 & -46.0 & 13 & 594 & Herbert et al 2016 & 182.42 & -47.85 & Alkenone \\
\hline 4.5 & 60.0 & 17.0 & 27.5 & 722 & Herbert et al 2016 & 59.09 & 15.78 & Alkenone \\
\hline 4.5 & 121.0 & 9.0 & 29 & 769 & Zhang et al 2014 & 120.36 & 9.04 & TEX86 \\
\hline 4.5 & 159.0 & 0.0 & 28 & 806 & Zhang et al 2014 & 162.14 & -1.64 & TEX86 \\
\hline 4.5 & -91.0 & -3.0 & 26.5 & 846 & Zhang et al 2014 & -93.57 & -3.33 & Alkenone \\
\hline 4.5 & -95.0 & 0.0 & 26.5 & 847 & Zhang et al 2014 & -97.49 & -0.23 & Alkenone \\
\hline 4.5 & -111.0 & 1.0 & 26.5 & 850 & Herbert et al 2016 & -107.86 & 0.01 & Alkenone \\
\hline 4.5 & -111.0 & 1.0 & 26.5 & 850 & Zhang et al 2014 & -107.86 & 0.01 & TEX86 \\
\hline 4.5 & -13.0 & 69.0 & 9 & 907 & Herbert et al 2016 & -13.90 & 69.03 & Alkenone \\
\hline 4.5 & -16.0 & 58.0 & 19 & 982 & Herbert et al 2016 & -15.38 & 58.02 & Alkenone \\
\hline 4.5 & -118.0 & 30.0 & 21.5 & 1010 & Herbert et al 2016 & -115.69 & 28.81 & Alkenone \\
\hline 4.5 & -128.0 & 39.0 & 17 & 1021 & Herbert et al 2016 & -125.85 & 37.56 & Alkenone \\
\hline 4.5 & 14.0 & -30.0 & 21 & 1085 & Herbert et al 2016 & 13.11 & -30.29 & Alkenone \\
\hline 4.5 & 14.0 & -41.0 & 18.5 & 1088 & Herbert et al 2016 & 13.03 & -41.29 & Alkenone \\
\hline 4.5 & -178.0 & -42.0 & 19 & 1125 & Herbert et al 2016 & -175.10 & -43.90 & Alkenone \\
\hline 4.5 & 113.0 & 9.0 & 29 & 1143 & Zhang et al 2014 & 112.36 & 9.06 & TEX86 \\
\hline 4.5 & 158.5 & 36.1 & 22 & 1208 & Herbert et al 2016 & 162.36 & 34.40 & Alkenone \\
\hline 4.5 & -86.0 & 6.0 & 28.2 & 1241 & Herbert et al 2016 & -88.37 & 3.09 & Alkenone \\
\hline 4.5 & -118.0 & 3.0 & 27.5 & U1338 & Herbert et al 2016 & -114.75 & 1.32 & Alkenone \\
\hline 4.5 & -118.0 & 3.0 & 27 & U1338 & Zhang et al 2014 & -114.75 & 1.32 & Alkenone \\
\hline 4.5 & 168.0 & 51.0 & 9 & $883 / 4$ & Herbert et al 2016 & 172.00 & 49.22 & Alkenone \\
\hline 4.5 & 14.0 & 44.0 & 25 & med & Herbert et al 2016 & 13.58 & 43.71 & Alkenone \\
\hline 7.5 & 175.0 & -46.0 & 16 & 594 & Herbert et al 2016 & 181.91 & -48.88 & Alkenone \\
\hline 7.5 & 60.0 & 17.0 & 28 & 722 & Herbert et al 2016 & 58.45 & 14.99 & Alkenone \\
\hline 7.5 & 159.0 & 0.0 & 30.5 & 806 & Zhang et al 2014 & 164.22 & -2.58 & TEX86 \\
\hline 7.5 & -91.0 & -3.0 & 28 & 846 & Herbert et al 2016 & -95.76 & -3.53 & Alkenone \\
\hline 7.5 & -111.0 & 1.0 & 27.6 & 850 & Herbert et al 2016 & -105.77 & -0.65 & Alkenone \\
\hline 7.5 & -111.0 & 1.0 & 27.5 & 850 & Zhang et al 2014 & -105.77 & -0.65 & TEX86 \\
\hline 7.5 & -148.0 & 54.0 & 16 & 887 & Herbert et al 2016 & -144.23 & 51.25 & Alkenone \\
\hline 7.5 & -13.0 & 69.0 & 11 & 907 & Herbert et al 2016 & -14.50 & 69.04 & Alkenone \\
\hline 7.5 & -16.0 & 58.0 & 23 & 982 & Herbert et al 2016 & -15.00 & 58.04 & Alkenone \\
\hline
\end{tabular}




\begin{tabular}{|c|c|c|c|c|c|c|c|c|}
\hline Age (Ma) & Longitude & Latitude & $\mathrm{T}(\operatorname{deg} C)$ & Site name & Reference & $\begin{array}{l}\text { Paleo } \\
\text { Latitude }\end{array}$ & $\begin{array}{l}\text { Paleo } \\
\text { longitude }\end{array}$ & Proxy \\
\hline 7.5 & -118.0 & 30.0 & 24 & 1010 & "Herbert et al 2016 & -114.05 & 28.18 & Alkenone \\
\hline 7.5 & -128.0 & 39.0 & 17 & 1021 & Herbert et al 2016 & -124.28 & 36.79 & Alkenone \\
\hline 7.5 & 14.0 & -30.0 & 23 & 1085 & Herbert et al 2016 & 12.53 & -30.46 & Alkenone \\
\hline 7.5 & 14.0 & -41.0 & 20 & 1088 & Herbert et al 2016 & 12.39 & -41.46 & Alkenone \\
\hline 7.5 & -178.0 & -42.0 & 23 & 1125 & Herbert et al 2016 & -173.08 & -44.94 & Alkenone \\
\hline 7.5 & 113.0 & 9.0 & 30 & 1143 & Zhang et al 2014 & 111.95 & 9.08 & TEX86 \\
\hline 7.5 & 158.5 & 36.1 & 24 & 1208 & Herbert et al 2016 & 164.81 & 33.36 & Alkenone \\
\hline 7.5 & -86.0 & 6.0 & 27.9 & 1241 & Herbert et al 2016 & -90.53 & 1.20 & Alkenone \\
\hline 7.5 & -118.0 & 3.0 & 28 & U1338 & Herbert et al 2016 & -112.59 & 0.71 & Alkenone \\
\hline 7.5 & -118.0 & 3.0 & 27 & U1338 & Zhang et al 2014 & -112.59 & 0.71 & Alkenone \\
\hline 7.5 & 168.0 & 51.0 & 12 & $883 / 4$ & Herbert et al 2016 & 174.49 & 48.21 & Alkenone \\
\hline 7.5 & 14.0 & 44.0 & 26 & med & Herbert et al 2016 & 13.33 & 43.53 & Alkenone \\
\hline 10 & 175.0 & -46.0 & 19.5 & 594 & Herbert et al 2016 & 180.29 & -49.58 & Alkenone \\
\hline 10 & -23.0 & 42.8 & 24 & 608 & Super et al 2018 & -24.62 & 42.75 & TEX86 \\
\hline 10 & 60.0 & 17.0 & 28 & 722 & Herbert et al 2016 & 57.85 & 14.28 & Alkenone \\
\hline 10 & 121.0 & 9.0 & 30 & 769 & Zhang et al 2014 & 119.58 & 9.11 & TEX86 \\
\hline 10 & 159.0 & 0.0 & 30 & 806 & Zhang et al 2014 & 165.92 & -3.22 & TEX86 \\
\hline 10 & -91.0 & -3.0 & 28.5 & 846 & Herbert et al 2016 & -97.89 & -3.68 & Alkenone \\
\hline 10 & -111.0 & 1.0 & 27.8 & 850 & Herbert et al 2016 & -104.03 & -1.20 & Alkenone \\
\hline 10 & -111.0 & 1.0 & 27.5 & 850 & Zhang et al 2014 & -104.03 & -1.20 & Alkenone \\
\hline 10 & -13.0 & 69.0 & 17 & 907 & Herbert et al 2016 & -15.00 & 69.06 & Alkenone \\
\hline 10 & -16.0 & 58.0 & 25 & 982 & Herbert et al 2016 & -14.62 & 58.01 & Alkenone \\
\hline 10 & -118.0 & 30.0 & 26 & 1010 & Herbert et al 2016 & -112.62 & 27.77 & Alkenone \\
\hline 10 & -128.0 & 39.0 & 21 & 1021 & Herbert et al 2016 & -122.89 & 36.28 & Alkenone \\
\hline 10 & 14.0 & -30.0 & 25 & 1085 & Herbert et al 2016 & 12.03 & -30.65 & Alkenone \\
\hline 10 & 14.0 & -41.0 & 20 & 1088 & Herbert et al 2016 & 11.85 & -41.65 & Alkenone \\
\hline 10 & -178.0 & -42.0 & 25 & 1125 & Herbert et al 2016 & -171.39 & -45.64 & Alkenone \\
\hline 10 & 113.0 & 9.0 & 30 & 1143 & Zhang et al 2014 & 111.62 & 9.11 & TEX86 \\
\hline 10 & 158.5 & 36.1 & 24 & 1208 & Herbert et al 2016 & 166.76 & 32.59 & Alkenone \\
\hline 10 & -86.0 & 6.0 & 28.2 & 1241 & Herbert et al 2016 & -92.68 & -0.35 & Alkenone \\
\hline 10 & -118.0 & 3.0 & 28 & U1338 & Herbert et al 2016 & -110.79 & 0.31 & Alkenone \\
\hline 10 & -118.0 & 3.0 & 27.5 & U1338 & Zhang et al 2014 & -110.79 & 0.31 & Alkenone \\
\hline 10 & 168.0 & 51.0 & 14 & $883 / 4$ & Herbert et al 2016 & 176.47 & 47.51 & Alkenone \\
\hline 10 & 14.0 & 44.0 & 28 & med & Herbert et al 2016 & 13.13 & 43.33 & Alkenone \\
\hline 12.5 & 175.0 & -46.0 & 23.5 & 594 & Herbert et al 2016 & 179.15 & -50.10 & Alkenone \\
\hline 12.5 & -23.0 & 42.8 & 27 & 608 & Super et al 2018 & -24.91 & 42.61 & TEX86 \\
\hline 12.5 & -91.0 & -3.0 & 28.5 & 846 & Herbert et al 2016 & -100.28 & -3.82 & Alkenone \\
\hline 12.5 & -111.0 & 1.0 & 28.5 & 850 & Herbert et al 2016 & -102.28 & -1.75 & Alkenone \\
\hline 12.5 & -13.0 & 69.0 & 19 & 907 & Herbert et al 2016 & -15.50 & 69.07 & Alkenone \\
\hline
\end{tabular}




\begin{tabular}{|c|c|c|c|c|c|c|c|c|}
\hline Age (Ma) & Longitude & Latitude & $\mathrm{T}(\operatorname{deg} C)$ & Site name & Reference & $\begin{array}{l}\text { Paleo } \\
\text { Latitude }\end{array}$ & $\begin{array}{l}\text { Paleo } \\
\text { longitude }\end{array}$ & Proxy \\
\hline 12.5 & $\begin{array}{l}-16.0 \\
\end{array}$ & 58.0 & 26 & 982 & Herbert et al 2016 & "-14.14 & 57.90 & Alkenone \\
\hline 12.5 & -118.0 & 30.0 & 27 & 1010 & Herbert et al 2016 & -111.12 & 27.45 & Alkenone \\
\hline 12.5 & -128.0 & 39.0 & 22 & 1021 & Herbert et al 2016 & -121.44 & 35.90 & Alkenone \\
\hline 12.5 & 14.0 & -30.0 & 27 & 1085 & Herbert et al 2016 & 11.51 & -30.92 & Alkenone \\
\hline 12.5 & 14.0 & -41.0 & 20 & 1088 & Herbert et al 2016 & 11.29 & -41.92 & Alkenone \\
\hline 12.5 & -118.0 & 3.0 & 28.3 & U1338 & Herbert et al 2016 & -108.98 & 0.00 & Alkenone \\
\hline 12.5 & 14.0 & 44.0 & 28 & med & Herbert et al 2016 & 12.95 & 43.05 & Alkenone \\
\hline 15 & -23.0 & 42.8 & 31 & 608 & Super et al 2018 & -25.07 & 42.33 & TEX86 \\
\hline 15 & -16.0 & 58.0 & 27 & 982 & Herbert et al 2016 & -13.51 & 57.66 & Alkenone \\
\hline 15 & -118.0 & 3.0 & 28.8 & U1338 & Herbert et al 2016 & -107.18 & -0.20 & Alkenone \\
\hline 15 & 149.0 & -48.0 & 17 & 1171 & Shevenell et al 2004 & 149.02 & -56.75 & $\delta^{18} \mathrm{O}$ \\
\hline 15 & 116.00 & 19.00 & 28 & 1146 & Holbourn et al 2010 & 114.01 & 19.01 & $\delta^{18} \mathrm{O}$ \\
\hline
\end{tabular}

Table S1, Surface ocean temperature used in this study estimated from published data, data points are those within a $\pm 1 \mathrm{million}$ year window around our target age.

\section{References for A}

Herbert, T.D., Lawrence, K.T., Tzanova, A., Peterson, L.C., Caballero-Gill, R. and Kelly, C.S.: Late Miocene global cooling and the rise of modern ecosystems, Nat. Geosci., 9(11), p.843, doi: 10.1038/ngeo2813, 2016.

Holbourn, A., Kuhnt, W., Regenberg, M., Schulz, M., Mix, A. and Andersen, N.: Does Antarctic glaciation force migration of the tropical rain belt?, Geology, 38(9), pp.783-786, doi:10.1130/G31043.1, 2010.

Shevenell, A.E., Kennett, J.P. and Lea, D.W.: Middle Miocene southern ocean cooling and Antarctic cryosphere expansion. Science, 305(5691), pp.1766-1770, doi:10.1126/science.1100061, 2004.

Super, J.R., Thomas, E., Pagani, M., Huber, M., O’Brien, C. and Hull, P.M.: North Atlantic temperature and p CO2 coupling in the early-middle Miocene. Geology, 46(6), pp.519-522, doi: 10.1130/G40228.1, 2018.

Zhang, Y.G., Pagani, M. and Liu, Z.: A 12-million-year temperature history of the tropical Pacific Ocean. Science, 344(6179), pp.84-87, doi: 10.1126/science.1246172, 2014. 


\section{Supplementary material B}

\section{Benthic ocean $\delta^{13} \mathrm{C}$ and $\delta^{18} \mathrm{O}$ data points}

\begin{tabular}{|c|c|c|c|c|c|c|c|c|c|c|}
\hline 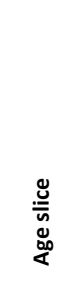 & 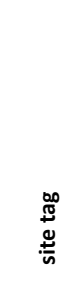 & 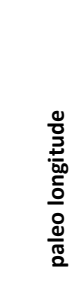 & 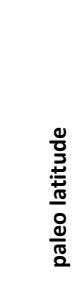 & $\begin{array}{l}\frac{5}{0} \\
\frac{0}{8}\end{array}$ & 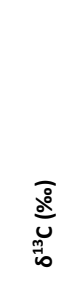 & 高 & 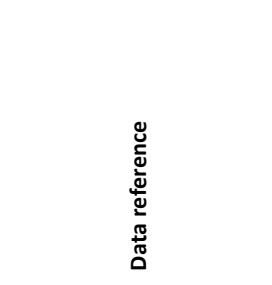 & 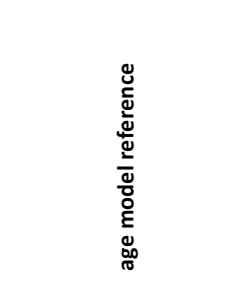 & 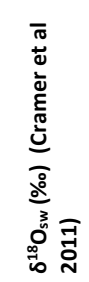 & 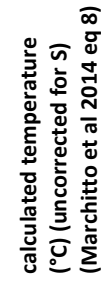 \\
\hline 15 & 608 & -25.1 & 42.4 & 3526 & 1.25 & 0.95 & Miller et al 1987 & Miller et al 1987 & -0.75 & 6.93 \\
\hline 15 & 1146 & 114.1 & 19.7 & 2092 & 1.4 & 1.4 & Holbourn et al 2007 & $\begin{array}{l}\text { Nathan and Leckie } \\
2003\end{array}$ & -0.75 & 4.93 \\
\hline 15 & U1336 & -118.3 & 4.5 & 4286 & 1.44 & 1.5 & Voigt et al 2015 & Voigt et al 2015 & -0.75 & 4.49 \\
\hline 15 & U1335 & -116.3 & 2.2 & 4330 & 1.25 & 1.6 & Kochhann et al 2016 & Kochhann et al 2016 & -0.75 & 4.04 \\
\hline 15 & 667 & -24.3 & 4.1 & 3529 & 1.3 & 1.95 & Woodruff and Savin 1991 & Ruddimann et al 2005 & -0.75 & 2.49 \\
\hline 15 & U1337 & -113.1 & 0.9 & 4211 & 1.35 & 1.4 & Kochhann et al 2016 & Kochhann et al 2016 & -0.75 & 4.93 \\
\hline 15 & U1338 & -107.6 & -0.2 & 4211 & 1.3 & 1.29 & Holbourn et al 2014 & Holbourn et al 2014 & -0.75 & 5.42 \\
\hline 15 & 806 & 169.5 & -3.5 & 2520 & 1.86 & 1.5 & Corfield et al 1993 & Corfield et al 1993 & -0.75 & 4.49 \\
\hline 15 & 289 & 168.7 & -4.3 & 2206 & 1.45 & 1.25 & Woodruff and Savin 1991 & Lazarus et al 1995 & -0.75 & 5.60 \\
\hline 15 & 709 & 57.9 & -5.4 & 3049 & 1.035 & 1.55 & Woodruff 1990 & Woodruff 1990 & -0.75 & 4.27 \\
\hline 15 & 237 & 55.4 & -8.4 & 1623 & 1.6 & 1.62 & Woodruff and Savin 1991 & $\begin{array}{l}\text { Woodruff and Savin } \\
1991\end{array}$ & -0.75 & 3.96 \\
\hline 15 & 525 & -0.3 & -30.2 & 2467 & 1.5 & 2.1 & Woodruff and Savin 1991 & Lazarus et al 1995 & -0.75 & 1.82 \\
\hline 15 & 1172 & 149.6 & -52.4 & 2622 & 1.52 & 1.75 & Ennyu et al 2004 & Ennyu et al 2004 & -0.75 & 3.38 \\
\hline 15 & 1170 & 145.3 & -55.9 & 2705 & 1.45 & 1.4 & Ennyu et al 2004 & Ennyu et al 2004 & -0.75 & 4.93 \\
\hline 15 & 744 & 77.6 & -61.4 & 2317 & 1.5 & 1.8 & Majewski 2002 & Majewski 2002 & -0.75 & 3.16 \\
\hline 12.5 & 555 & -22.9 & 56.4 & 1582 & 1 & 1.9 & $\begin{array}{l}\text { Wright Miller Fairbanks } \\
1992\end{array}$ & Cramer et al 2018 & -0.2 & 5.16 \\
\hline 12.5 & 553 & -25.4 & 55.9 & 2233 & 1 & 1.8 & Miller Aubry et al 1985 & Cramer et al 2018 & -0.2 & 5.60 \\
\hline 12.5 & 608 & -25.0 & 42.7 & 3379 & 0.95 & 1.8 & $\begin{array}{l}\text { Wright Miller 1996, Miller } \\
\text { Fairbanks Thomas } 1987\end{array}$ & Cramer et al 2018 & -0.2 & 5.60 \\
\hline 12.5 & 563 & -41.9 & 33.4 & 3518 & 1.1 & 2.03 & $\begin{array}{l}\text { Woodruff and Savin 1991, } \\
\text { Wright Millar Fairbanks } \\
\text { 1991, Wright Millar } \\
\text { Fairbanks 1992, Miller } \\
\text { Fairbanks 1985 }\end{array}$ & Cramer et al 2018 & -0.2 & 4.56 \\
\hline 12.5 & 1146 & 114.5 & 19.6 & 2092 & 0.65 & 2.1 & Holbourn et al 2013 & Holbourn et al 2013 & -0.2 & 4.27 \\
\hline 12.5 & 667 & -23.8 & 4.3 & 3400 & 1 & 1.7 & Woodruff and Savin 1991 & Cramer et al 2018 & -0.2 & 6.04 \\
\hline 12.5 & 574 & -124.7 & 0.9 & 4268 & 0.9 & 2.4 & $\begin{array}{l}\text { Pisias Shackelton Hall } \\
1985\end{array}$ & Cramer et al 2018 & -0.2 & 2.93 \\
\hline 12.5 & 926 & -41.3 & 3.8 & 3626 & 1.2 & 1.75 & Shackelton Hall 1997 & Cramer et al 2018 & -0.2 & 5.82 \\
\hline 12.5 & 806 & 167.8 & -3.2 & 2520 & 1 & 2.1 & Corfield et al 1993 & Corfield et al 1993 & -0.2 & 4.27 \\
\hline 12.5 & 289 & 167.0 & -4.0 & 2453 & 0.9 & 2.25 & $\begin{array}{l}\text { Savin Douglas et al 1981, } \\
\text { Woodruff et al 1981, } \\
\text { Woodruff and Savin } 1991\end{array}$ & Cramer et al 2018 & -0.2 & 3.60 \\
\hline 12.5 & 709 & 58.4 & -5.0 & 3049 & 0.92 & 2.56 & Woodruff 1990 & Woodruff 1990 & -0.2 & 2.22 \\
\hline 12.5 & 237 & 56.0 & -8.0 & 1623 & 0.93 & 2.28 & Woodruff and Savin 1991 & $\begin{array}{l}\text { Woodruff and Savin } \\
1991\end{array}$ & -0.2 & 3.47 \\
\hline 12.5 & 317 & -153.5 & -15.2 & 2638 & 0.85 & 2.35 & Woodruff and Savin 1989 & Cramer et al 2018 & -0.2 & 3.16 \\
\hline 12.5 & 1237 & -86.2 & -17.8 & 3212 & 0.9 & 2.4 & Holbourn et al 2007 & Holbourn 2005 & -0.2 & 2.93 \\
\hline 12.5 & 527 & -0.8 & -28.8 & 4357 & 1 & 2.25 & $\begin{array}{l}\text { "ref 2" (Cramer et al } \\
\text { 2018) }\end{array}$ & Cramer et al 2018 & -0.2 & 3.60 \\
\hline 12.5 & 525 & 0.3 & -29.8 & 2470 & 1.5 & 2.2 & $\begin{array}{l}\text { Shackelton Hall and } \\
\text { Boersma } 1984\end{array}$ & Cramer et al 2018 & -0.2 & 3.82 \\
\hline 12.5 & 357 & -33.4 & -29.8 & 2037 & 1.1 & 2.4 & Savin & Cramer et al 2018 & -0.2 & 2.93 \\
\hline
\end{tabular}




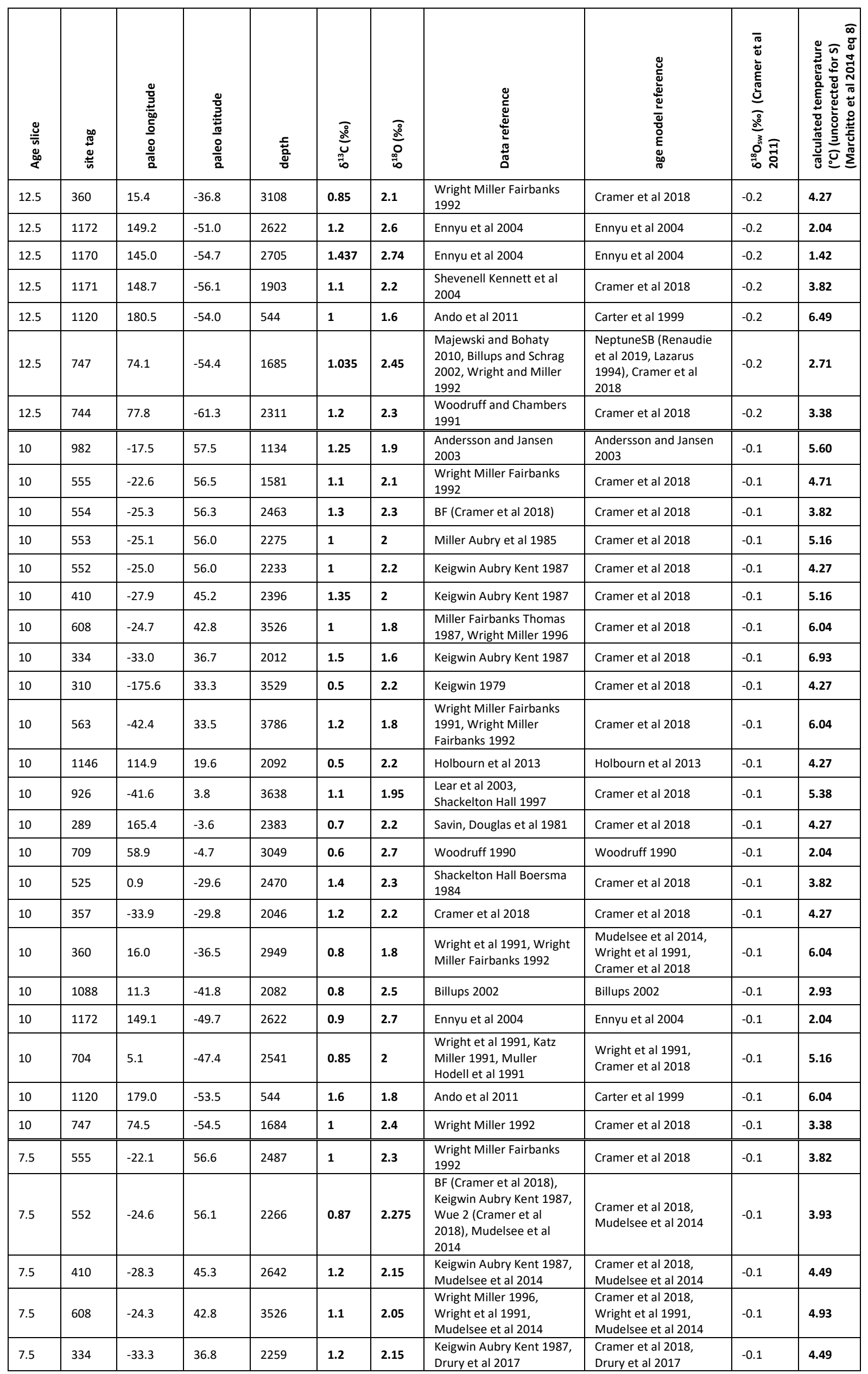




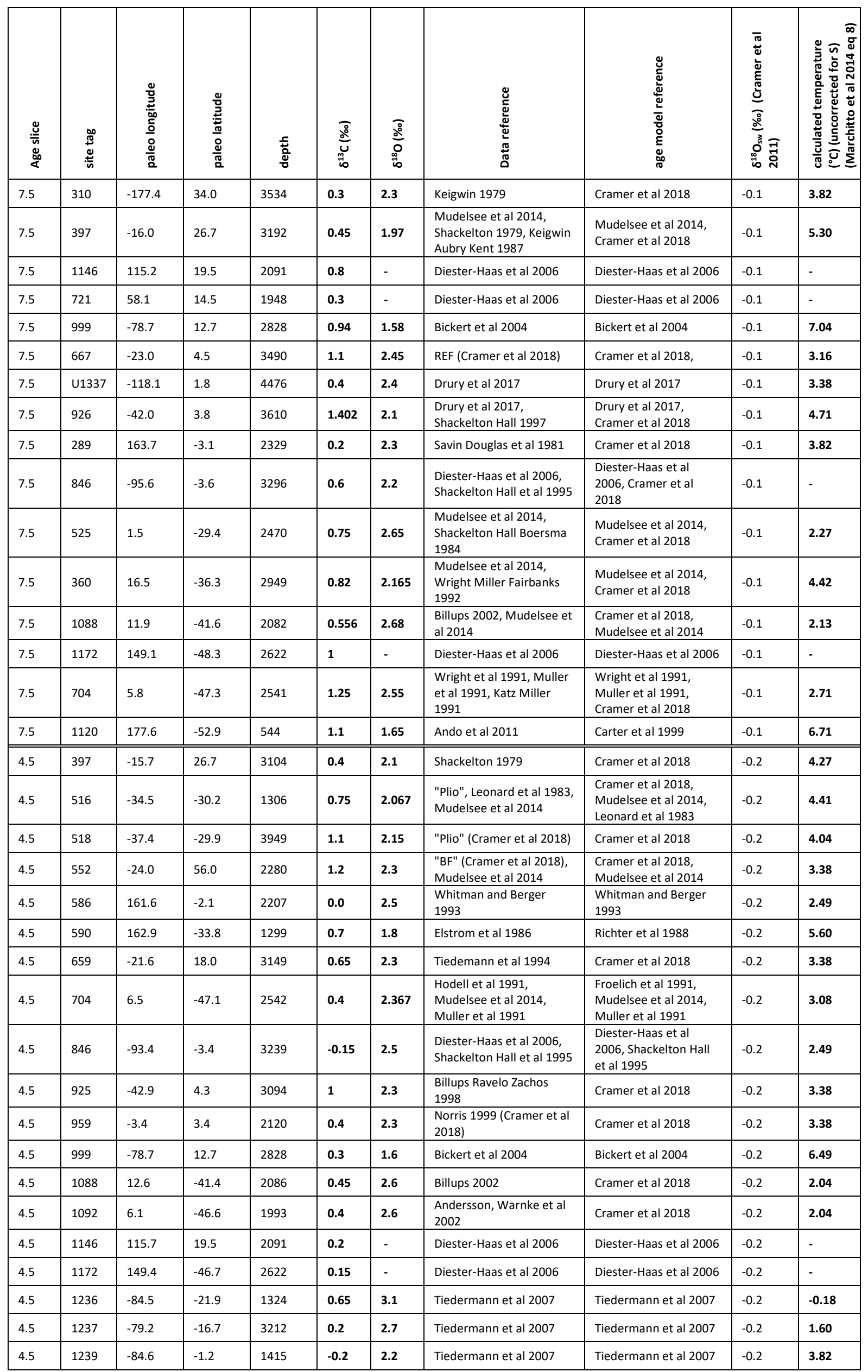




\begin{tabular}{|c|c|c|c|c|c|c|c|c|c|c|}
\hline 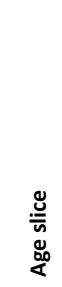 & $\begin{array}{l}\text { D0 } \\
\stackrel{0}{ \pm} \\
\stackrel{ \pm}{n}\end{array}$ & 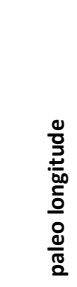 & 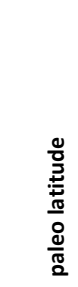 & $\begin{array}{l}\frac{5}{2} \\
\frac{0}{0}\end{array}$ & 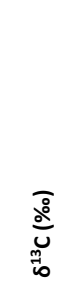 & 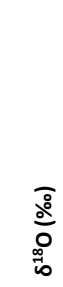 & 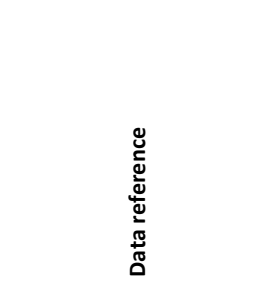 & 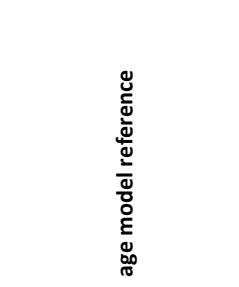 & 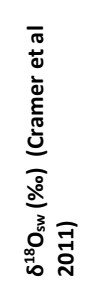 & 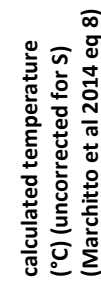 \\
\hline 4.5 & 1241 & -88.8 & 3.0 & 2027 & 0.1 & 2.4 & Tiedermann et al 2007 & Tiedermann et al 2007 & -0.2 & 2.93 \\
\hline 2.5 & 659 & -21.4 & 18.0 & 3115 & 0.35 & 3 & Tiedemann et al 1994 & Cramer et al 2018 & 0.05 & 1.38 \\
\hline 2.5 & 677 & -22.3 & 4.6 & 3352 & -0.5 & 3 & Shackelton Hall 1989 & Cramer et al 2018 & 0.05 & 1.38 \\
\hline 2.5 & 704 & 6.9 & -47.0 & 2543 & 0.2 & 3.2 & $\begin{array}{l}\text { Mudelsee et al 2014, } \\
\text { Hodell et al } 1991\end{array}$ & $\begin{array}{l}\text { Mudelsee et al 2014, } \\
\text { Froelich et al } 1991\end{array}$ & 0.05 & 0.49 \\
\hline 2.5 & 747 & 76.2 & -54.7 & 1701 & - & 3.5 & Billups Schrag 2002 & Cramer et al 2018 & 0.05 & -0.84 \\
\hline 2.5 & 806 & 161.1 & -0.7 & 2556 & - & 2.7 & $\begin{array}{l}\text { Lear Rosenthal Wright } \\
2003\end{array}$ & Cramer et al 2018 & 0.05 & 2.71 \\
\hline 2.5 & 846 & -92.1 & -3.3 & 3237 & -0.5 & 3 & Le Mix Shackleton 1995 & Cramer et al 2018 & 0.05 & 1.38 \\
\hline 2.5 & 1088 & 13.0 & -41.3 & 2081 & 0.5 & 3 & $\begin{array}{l}\text { Diester-Hass et al 2006, } \\
\text { Billups } 2002\end{array}$ & $\begin{array}{l}\text { Diester-Hass et al } \\
2006, \text { Billups } 2002\end{array}$ & 0.05 & 1.38 \\
\hline 2.5 & 1090 & 8.9 & -42.9 & 3720 & 0.1 & 3.1 & $\begin{array}{l}\text { Mudelsee et al 2014, } \\
\text { Hodell Charles et al } 2003\end{array}$ & $\begin{array}{l}\text { Mudelsee et al 2014, } \\
\text { Cramer et al } 2018\end{array}$ & 0.05 & 0.93 \\
\hline 2.5 & 1236 & -83.0 & -21.7 & 1323 & 0.3 & 3.5 & Tiedermann et al 2007 & Tiedermann et al 2007 & 0.05 & -0.84 \\
\hline 2.5 & 1239 & -83.4 & -1.0 & 3212 & -0.2 & 2.7 & Tiedermann et al 2007 & Tiedermann et al 2007 & 0.05 & 2.71 \\
\hline 2.5 & 1241 & -87.6 & 4.2 & 2027 & -0.3 & 3 & Tiedermann et al 2007 & Tiedermann et al 2007 & 0.05 & 1.38 \\
\hline 2.5 & 1313 & -33.0 & 41.0 & 3426 & 0.4 & - & Lang et al 2014 & Lang et al 2014 & 0.05 & - \\
\hline 2.5 & 397 & -15.5 & 26.8 & 2900 & 0.0 & 2.55 & $\begin{array}{l}\text { Mudelsee et al 2014, } \\
\text { Shackleton 1979, Stein } \\
1985\end{array}$ & $\begin{array}{l}\text { Mudelsee et al 2014, } \\
\text { Cramer et al } 2018\end{array}$ & 0.05 & 3.38 \\
\hline 2.5 & 516 & -34.9 & -30.2 & 1313 & 0.7 & 2.5 & Leonard et al 1983 & Leonard et al 1983 & 0.05 & 3.60 \\
\hline 2.5 & 518 & -37.7 & -29.9 & 3951 & 0.5 & 2.9 & "Plio" Cramer et al 2018 & Cramer et al 2018 & 0.05 & 1.82 \\
\hline 2.5 & 552 & -23.7 & 56.0 & 2287 & 0.7 & 3 & $\begin{array}{l}\text { Mudelsee et al 2014, "BF" } \\
\text { (Cramer et al 2018) }\end{array}$ & $\begin{array}{l}\text { Mudelsee et al 2014, } \\
\text { Cramer et al } 2018\end{array}$ & 0.05 & 1.38 \\
\hline 2.5 & 586 & 160.3 & -1.4 & 2207 & -0.2 & 3 & $\begin{array}{l}\text { Whitman and Berger } \\
1993\end{array}$ & $\begin{array}{l}\text { Whitman and Berger } \\
1993\end{array}$ & 0.05 & 1.38 \\
\hline 2.5 & 590 & 163.1 & -32.6 & 1299 & 0.7 & 2.6 & Elstrom et al 1986 & Richter et al 1988 & 0.05 & 3.16 \\
\hline 2.5 & 607 & -33.0 & 41.0 & 3407 & 0.8 & 2.8 & $\begin{array}{l}\text { Raymo Ruddiman et al } \\
1989\end{array}$ & Cramer et al 2018 & 0.05 & 2.27 \\
\hline
\end{tabular}

Table S2, Published benthic $\delta^{13} \mathrm{C}$ and $\delta^{18} \mathrm{O}$ data used in this study, together with calculated benthic temperature derived from $\delta^{18} \mathrm{O}$, Cramer et al. (2011) global ice volume, and the Marchitto et al. (2014) paleotemperature calculation (see main text) so is uncorrected for salinity in this table.

\section{References for B}

Andersson, C., Warnke, D.A., Channell, J.E.T., Stoner, J. and Jansen, E.: The mid-Pliocene (4.3-2.6 Ma) benthic stable isotope record of the Southern Ocean: ODP Sites 1092 and 704, Meteor Rise. Palaeogeogr. Palaeocli., 182(3-4), 165-181, doi: 10.1016/S0031-0182(01)00494-1, 2002.

Andersson, C., Jansen, E.: Miocene stable isotope record of ODP Hole 162-982B. PANGAEA, https://doi.org/10.1594/PANGAEA.842276, 2003.

Ando, A., Khim, B., Nakano, T., Takata, H.: Appendix A Table SD1. CaCO3, d180, d13C and Sr isotopes from ODP Site 181-1120. PANGAEA, https://doi.org/10.1594/PANGAEA.763934, 2011.

Bickert, T., Haug, G. H., Tiedemann, R.: Late Neogene benthic stable isotope record of ODP Hole 165-999A. PANGAEA, https://doi.org/10.1594/PANGAEA.840843, 2004 
Billups, K., and Schrag, D.P.: Paleotemperatures and ice volume of the past 27 Myr revisited with paired Mg/Ca and 180/160 measurements on benthic foraminifera, Paleoceanography, 17(1), 1003, doi:10.1029/2000PA000567, 2002.

Billups, K., Ravelo, A.C. and Zachos, J.C.: Early Pliocene deep water circulation in the western equatorial Atlantic: Implications for high-latitude climate change. Paleoceanography, 13(1), 84-95, doi: 10.1029/97PA02995, 1998.

Billups, K.: (Appendix I) Stable carbon and oxygen isotope ratios of Cibicidoides spp. from ODP Site 177-1088 in the subAntarctic South Atlantic. PANGAEA, https://doi.org/10.1594/PANGAEA.704454, 2002

Carter, R.M., McCave, I.N., Richter, C., Carter, L., et al., Proceedings of the Ocean Drilling Program, Initial Reports Volume 181, chpt 4 Site 1120 : Central Campbell Plateau. 1999.

Corfield, R. M., Cartlidge, J. E.: (Appendix B) Stable carbon and oxygen isotope ratios of bulk rock samples and foraminifera of ODP Hole 130-806B. PANGAEA, https://doi.org/10.1594/PANGAEA.780105, 1993.

Cramer, B. S.: (Data Set S1) Compiled benthic foraminiferal stable isotopic values. PANGAEA, https://doi.org/10.1594/PANGAEA.890185, 2018

Diester-Haass, L., Billups, K., Emeis, K.: Late Miocene carbon isotope and paleoproductivity record. PANGAEA, https://doi.org/10.1594/PANGAEA.835082, 2006.

Drury, AJ., Westerhold, T., Frederichs, T., Tian, J., Wilkens, R. H., Channell, J. E. T., Evans, H. F., John, C., M., Lyle, M. W., Röhl, U.,: (Supplement Table S6) Stable isotopes of ODP Site 154-926. PANGAEA https://doi.org/10.1594/PANGAEA.872509, 2017

Elmstrom, K. M., Kennett, J. P.: (Table 2) Planktonic and benthic foraminiferal oxygen and carbon isotopes at DSDP Holes 90-590A and 90-590B. PANGAEA, https://doi.org/10.1594/PANGAEA.793187, 1986.

Ennyu, A., Arthur, M. A.: (Table T1) Oxygen and carbon stable isotopes of calcareous microfossils from ODP Holes 1891170A and 189-1172A. PANGAEA, https://doi.org/10.1594/PANGAEA.780277, 2004.

Froelich, P. N., Malone, P. N, Hodell, D. A., Ciesielski, P. F., Warnke, D. A, Westall, F., Hailwood, E. A., Nobes, D. C., Fenner, J. M., Mienert, J., Mwenifumbo, C. J., Müller, D. W.: (Table 3) Chronology of the Site 114-704 composite section. PANGAEA, https://doi.org/10.1594/PANGAEA.754797, 1991.

Hodell, D.A., Charles, C.D., Curtis, J.H., Mortyn, P.G., Ninnemann, U.S., Venz, K.A.: Stable isotope record of benthic and planktonic foraminifera in sediment cores of ODP Leg 177, Southern Ocean. PANGAEA, https://doi.org/10.1594/PANGAEA.701432, 2003.

Hodell, D.A., Ciesielski, P.F.: Stable isotopic record of late Pliocene and Pleistocene foraminifera of ODP Site 114-704. PANGAEA, https://doi.org/10.1594/PANGAEA.754619, 1991.

Holbourn, A., Kuhnt, W., Clemens, S.C., Prell, W.L., Andersen, N.: Middle to late Miocene stable isotope record of benthic foraminifera from ODP Site 184-1146. PANGAEA, https://doi.org/10.1594/PANGAEA.825480, 2013.

Holbourn, A., Kuhnt, W., Lyle, M.W., Schneider, L., Romero, O.E., Andersen, N.: Stable isotope record, opal accumulation rate and geochemistry of middle Miocene sediments of IODP Site 321-U1338. PANGAEA, https://doi.org/10.1594/PANGAEA.820095, 2014

Holbourn, A., Kuhnt, W., Schulz, M., Erlenkeuser, H.: Geochemistry and stable isotope record of benthic foraminifera of Miocene sediments. PANGAEA, https://doi.org/10.1594/PANGAEA.738246, 2005

Holbourn, A., Kuhnt, W., Schulz, M., Flores, JA., Andersen, N.: Stable isotopes measured on Miocene benthic foraminifera from ODP sites 202-1237 and 184-1146. PANGAEA, https://doi.org/10.1594/PANGAEA.658956, 2007. 
Katz, M. E., Miller, K. G.: Benthic foraminiferal stable isotope record of the early Paleogene in the Southern Ocean. PANGAEA, https://doi.org/10.1594/PANGAEA.754016, 1991.

Keigwin, L. D.: Late Cenozoic stable isotope record of benthic and planktonic foraminifera from the Pacific Ocean. PANGAEA, https://doi.org/10.1594/PANGAEA.710854, 1979.

Keigwin, L. D., Aubry, MP., Kent, D.V.: (Appendix) Stabile isotopes $\delta^{18} \mathrm{O}$ and $\delta^{13} \mathrm{C}$ for DSDP Sites 37-334, 49-408, and 49410 and Holes 81-552A, 94-610E, and 94-611C. PANGAEA, https://doi.org/10.1594/PANGAEA.788680, 1987.

Kochhann, K.G.D., Holbourn, A., Kuhnt, W., Channell, J.E.T., Lyle, M.W. : Miocene stable isotope record of IODP Site 320U1335 and revised stable isotope record of IODP Site 321-U1337. PANGAEA, https://doi.org/10.1594/PANGAEA.863701, 2016.

Lang, D.C., Bailey, I., Wilson, P.A., Beer, C.J., Bolton, C.T., Friedrich, O., Newsam, C., Spencer, M.R., Gutjahr, M., Foster, G.L., Cooper, M.J., Milton, J.A.: Stable carbon isotope record of IODP Site 306-U1313. PANGAEA, https://doi.org/10.1594/PANGAEA.829424, 2014.

Lazarus, D. B.: Neptune: A marine Micropaleontology Database. Math. Geol., 26(7): 817-832, doi: 10.1007/BF02083119, 1994.

Lazarus, D.B., Spencer-Cervato, C., Pika-Biolzi, M., Beckmann, JP., von Salis, K.H., Hilbrecht, H., Thierstein, H.R.: Revised chronology of Neogene DSDP holes from the World Ocean. PANGAEA, https://doi.org/10.1594/PANGAEA.730465, 1995.

Le, J., Mix, A., and Shackleton, N.J.: Late Quaternary paleoceanography in the eastern equatorial Pacific from planktonic foraminifera: A high-resolution record from ODP Site 846. In: Pisias, N.G., L. Mayer, T. Janecek, A. Palmer-Julson, T.H. van Andel (eds.), Proceedings of the Ocean Drilling Program, /Scientific Results/ 138, College Station, TX (Ocean Drilling Program), 675-694, 1995.

Lear, C. H., Rosenthal, Y., and Wright, J.D. : The closing of a seaway: Ocean water masses and global climate change, Earth Planet. Sci. Lett., 210, 425-436, doi:10.1016/S0012-821X(03)00164-X, 2003.

Leonard, K.A., Williams, D.F., Thunell, R.C.: (Table 1) Oxygen and carbon isotopes of Cibicides wuellerstorfi and Cibicides kullenbergi at DSDP Hole 72-516A. PANGAEA, https://doi.org/10.1594/PANGAEA.812143, 1983.

Majewski, W.: (Appendix A) Stable isotope ratios of Cibicidoides sp. and planktonic foraminifera from the Mid-Miocene of ODP Hole 119-744B of the Kerguelen Plateau, Antarctica. PANGAEA, https://doi.org/10.1594/PANGAEA.678476, 2002.

Majewski, W., Bohaty, S.M.: Middle Miocene stable carbon and oxygen isotope record of foraminifera of ODP Hole 120747A. PANGAEA, https://doi.org/10.1594/PANGAEA.775084, 2010.

Miller, K.G. and Fairbanks, R.G. : Oligocene to Miocene carbon isotope cycles and abyssal circulation changes. In: Sundquist ET, Broecker WS (eds) The Carbon Cycle and Atmospheric CO2: Natural Variations Archean to Present, Tarpon Springs, Washington, 469-486, doi: 10.1029/GM032p0469, 1985.

Miller, K.G., Aubry, M.P., Khan, M.J., Melillo, A.J., Kent, D.V. and Berggren, W.A. : Oligocene-Miocene biostratigraphy, magnetostratigraphy, and isotopic stratigraphy of the western North Atlantic. Geology, 13(4), 257-261, doi : 10.1130/0091-7613(1985)13<257:OBMAIS>2.0.CO;2, 1985.

Miller, K.G., Fairbanks, R.G., Thomas, E.: (Table 2) Oxygen and carbon isotopic values for mixed species of Cibicidoides, DSDP Hole 94-608. PANGAEA, https://doi.org/10.1594/PANGAEA.788711, 1987. 
Miller, K.G., Fairbanks, R.G., Thomas, E.: Benthic foraminiferal carbon isotopic records and the development of abyssal circulation at DSDP Sites 9-77, 12-119, 30-289, 82-563 and 94-608. PANGAEA, https://doi.org/10.1594/PANGAEA.788720, 1987.

Mudelsee, M., Bickert, T., Lear, C.H., Lohmann, G.: Oxygen isotopic values for benthic foraminifera from DSDP and ODP high latitude marine sediment cores. PANGAEA, https://doi.org/10.1594/PANGAEA.844171, 2014.

Müller, D.W., Hodell, D.A., Ciesielski, P.F.: (Table 2) Stable isotopicratios of benthic foraminifera from ODP Hole 114704B. PANGAEA, https://doi.org/10.1594/PANGAEA.754683, 1991.

Nathan, S.A., Leckie, R.M.: Planktonic foraminifera stratigraphy and datums of ODP Site 184-1143 and Hole 184-1146A, South China Sea. PANGAEA, https://doi.org/10.1594/PANGAEA.784361, 2003.

Pisias, N.G., Shackleton, N.J., Hall, M.A.: (Table 1) Stable isotope ratios of benthic foraminifera from DSDP Hole 85-574A. PANGAEA, https://doi.org/10.1594/PANGAEA.803485, 1985.

Raymo, M.E., Ruddiman, W.F., Backman, J., Clement, B.M., Martinson, D.G.: Stable isotope record and calcium carbonate concentrations of Late Pliocene sediments of the North Atlantic. PANGAEA, https://doi.org/10.1594/PANGAEA.701337, 1989.

Renaudie, J., Lazarus, D.B., Diver P.: NSB: an expanded and improved database of marine planktonic microfossil data and deep-sea stratigraphy. Available as a preprint on EarthArXiv. 2019.

Richter, F.M., DePaolo, D.J.: Sr isotopic data from DSDP Sites 575 and 590. PANGAEA, https://doi.org/10.1594/PANGAEA.700902, 1988.

Ruddiman, W.F., Sarnthein, M., Shipboard Scientific Party: Depth-age model of ODP Hole 108-667A. PANGAEA, https://doi.org/10.1594/PANGAEA.797755, 2005.

Savin, S.M., Douglas, R.G., Keller, G., Killingley, J.S., Shaughnessy, L., Sommer, M.A., Vincent, E. and Woodruff, F.: Miocene benthic foraminiferal isotope records: a synthesis. Mar. Micropaleontol., 6(4), 423-450., doi: 10.1016/0377-8398(81)90031-1, 1981.

Shackelton, N.J. and Cita, M.B.: Oxygen and carbon isotope stratigraphy of benthic foraminifers at site 397: Detailed history of climatic changes during the Neogene. Init. Rep. DSDP, 47, 433-445. 1979.

Shackleton, N.J. and Hall, M.A.: 24. The late Miocene stable isotope record, Site 926. In Proceedings of the Ocean Drilling Program, Sci. Results, 154, 367-373, 1997.

Shackleton, N.J., Hall, M.A.: Stable carbon and oxygen isotope record of planktonic and benthic foraminifera at ODP Site 111-677 in the Panama Basin. PANGAEA, https://doi.org/10.1594/PANGAEA.701316, 1989.

Shackleton, N.J, Hall, M.A., Boersma, A.: Oxygen and carbon isotopes from benthic and planktonic foraminifers at DSDP Leg 74 Holes. PANGAEA, https://doi.org/10.1594/PANGAEA.810383, 1984.

Shackleton, N.J., Hall, M.A., Pate, D.: Pliocene stable oxygen and carbon isotope record of benthic foraminifera from ODP Site 138-846 in the eastern equatorial Pacific. PANGAEA, https://doi.org/10.1594/PANGAEA.696450, 1995.

Shevenell, A.E. and Kennett, J.P. : Paleoceanographic change during the middle Miocene climate revolution: an Antarctic stable isotope perspective. The Cenozoic Southern Ocean: Tectonics, Sedimentation, and Climate Change Between Australia and Antarctica, Geophys. Monogr. Ser, 151, 235-252, doi: 10.1029/151GM14, 2004.

Stein, R.: Late Neogene changes of paleoclimate and paleoproductivity off northwest Africa (DSDP Site 397). Palaeogeogr. Palaeocl., 49, 47-59, doi: 10.1016/0031-0182(85)90004-5, 1985.

Tiedemann, R., Sarnthein, M., Shackleton, N.J.: Benthic oxygen isotope and dust flux record from ODP Site 108-659 in the North Atlantic. PANGAEA, https://doi.org/10.1594/PANGAEA.696121, 1994. 
Tiedemann, R., Sturm, A., Steph, S., Lund, S.P., Stoner, J.S.: Age models and foraminifer isotopes of four ODP Leg 202 sites. PANGAEA, https://doi.org/10.1594/PANGAEA.774009, 2007.

Voigt, J., Hathorne, E.C., Frank, M., Holbourn, A.: Miocene stable isotope record of benthic foraminifera from IODP Site 320-U1336. PANGAEA, https://doi.org/10.1594/PANGAEA.854835, 2015.

Whitman, J.M., Berger, W.H.: (Appendix C) Stable carbon isotope ratios of foraminifera at DSDP Site 89-586. PANGAEA, https://doi.org/10.1594/PANGAEA.780396, 1993.

Whitman, J.M., Berger, W.H.: (Appendix) Stable oxygen isotope ratios of foraminifera at DSDP Site 89-586 on the Ontong Java Plateau, Pacific Ocean. PANGAEA, https://doi.org/10.1594/PANGAEA.683835, 1992.

Woodruff, F. and Douglas, R.G.: Response of deep-sea benthic foraminifera to Miocene paleoclimatic events, DSDP Site 289. Mar. Micropaleontol., 6(5-6), 617-632, doi: 10.1016/0377-8398(81)90024-4 1981.

Woodruff, F. and Savin, S.M.: Miocene deepwater oceanography. Paleoceanography, 4(1), 87-140, doi: 10.1029/PA004i001p00087, 1989.

Woodruff, F., Chambers, S.R.: Benthic foraminifer $\mathrm{d} 180$ and $\mathrm{d} 13 \mathrm{C}$ values and planktonic to benthic foraminifer ratios for ODP Hole 119-744B (Table 1). PANGAEA, https://doi.org/10.1594/PANGAEA.758064, 1991.

Woodruff, Fay; Savin, Samuel M (1991): Age models and stable isotope ratios from the Mid-Miocene. PANGAEA, https://doi.org/10.1594/PANGAEA.704918, 1991.

Woodruff, F., Savin, S.M., Abel, L.: Miocene benthic foraminifer oxygen and carbon isotope record of ODP Site 115-709. PANGAEA, https://doi.org/10.1594/PANGAEA.755792, 1990.

Wright, J. D., and K. G. Miller: Control of North Atlantic Deep Water circulation by the Greenland-Scotland Ridge, Paleoceanography, 11(2), 157-170, doi:10.1029/95PA03696, 1996.

Wright, J.D., Miller, K.G.: Miocene stable isotope stratigraphy of ODP Hole 120-747A. PANGAEA, https://doi.org/10.1594/PANGAEA.758917, 1992.

Wright, J.D., Miller, K.G., Fairbanks, R.G.: Stable isotope record of Cibicidoides spp. from Late Miocene sediments of the Southern Ocean. PANGAEA, https://doi.org/10.1594/PANGAEA.733982, 1991.

Wright, J.D., Miller, K.G., Fairbanks, R.G.: Stable isotope record of Cibicidoides spp. from early and middle Miocene sediments. PANGAEA, https://doi.org/10.1594/PANGAEA.728102, 1992. 


\section{Supplementary material C}

\section{Additional figures}
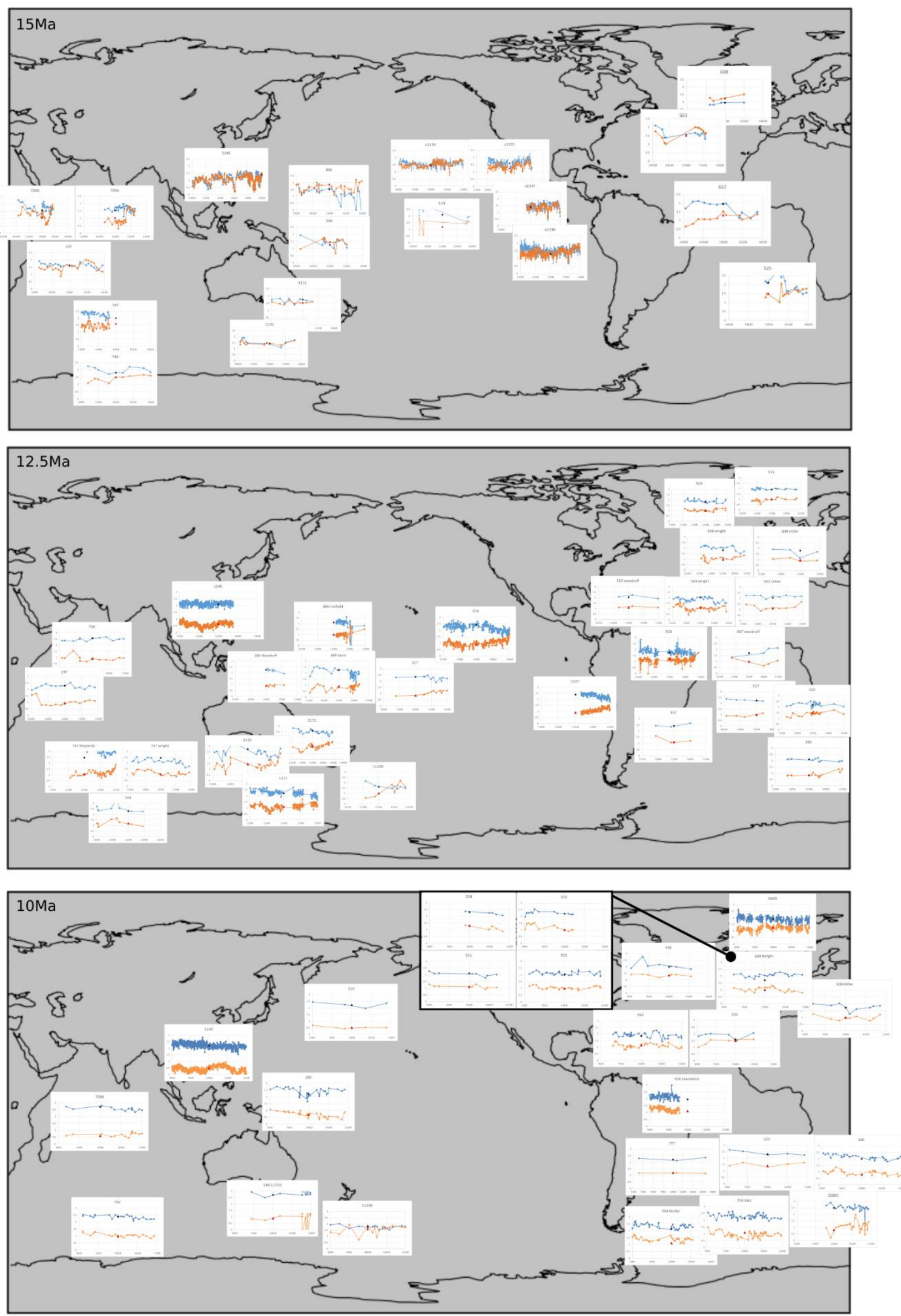

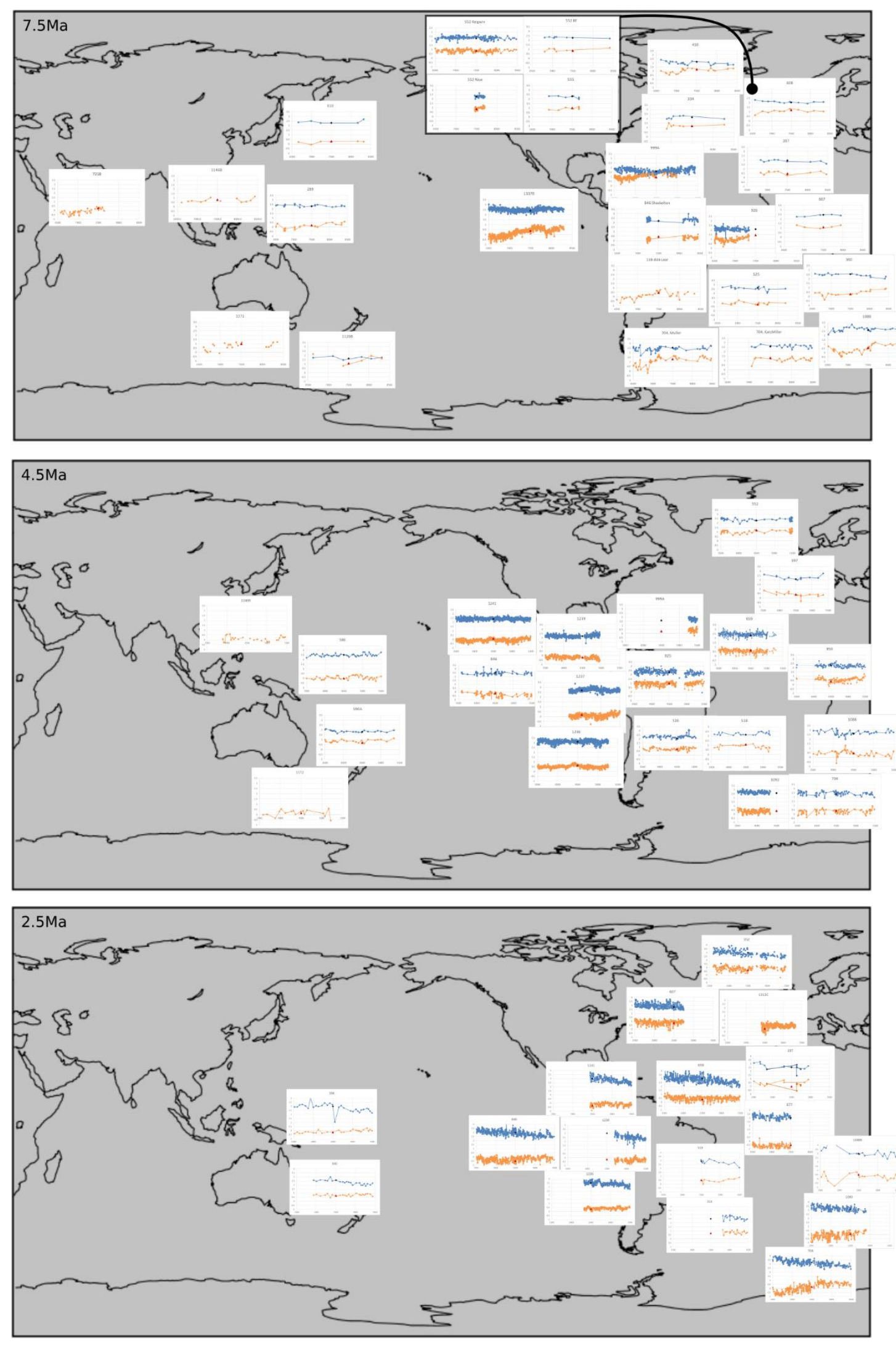

Figure S1, Datamaps for each time slice indicating the (approximate) location of the sediment core in the present day, and $\pm 1 \mathrm{Ma}$ window around the target age for $\delta^{13} \mathrm{C}$ and $\delta^{18} \mathrm{O}$. Target age is marked top-left on each map. Data points corresponds to that in Appendix B (black filled circle for $\delta^{18} \mathrm{O}$, red filled triangle for $\delta^{13} \mathrm{C}$ ). Where more than one record is available for a core site, the higher resolution set or that from Cramer et al. (2018) is plotted here. All data is plotted permil (\%o), $\delta^{18} \mathrm{O}$ timeseries coloured blue, $\delta^{13} \mathrm{C}$ is orange. 